\title{
Ventricular pseudoaneurysm rupture - a potentially fatal complication of myocardial infarction
}

\author{
Helena Krysztofiak', Jacek Migaj ${ }^{1}$, Piotr Buczkowski², Sebastian Stefaniak ${ }^{2}$, Ewa Straburzyńska-Migaj ${ }^{1}$, \\ Marta Kałużna-Oleksy ${ }^{1}$ \\ ${ }^{1} 1^{\text {st }}$ Department of Cardiology, Poznan University of Medical Sciences, Poznan, Poland \\ ${ }^{2}$ Clinic of Cardiac Surgery, Poznan University of Medical Science, Poznan, Poland
}

Adv Interv Cardiol 2020; 16, 4 (62): 512-513

DOI: https://doi.org/10.5114/aic.2020.101781

A 75-year-old hypertensive female patient presented at the cardiac department because of chest pain 20 days earlier. A suspicion of a past myocardial infarction (MI) and a pseudoaneurysm developed after echocardiography performed in the admissions office. Coronary angiography showed all vessels patent apart from the occluded left circumflex artery (LCX) (Figure $1 \mathrm{~A}$ ). Echocardiography showed pseudoaneurysm of the lateral wall of the left ventricle (LV) and small pericardial effusion (Figure $1 \mathrm{~B}$ ). Clinical status of the patient was stable. Cardiac magnetic resonance (CMR) was performed to rule out other cardiac pathologies - the free heart wall rupture was visualized, and consequently left ventricular pseudoaneurysm was confirmed (Figures 1 C, D). There were no abnormal laboratory results, and biomarkers of myocardial necrosis were negative. The patient was scheduled for urgent cardiac surgery. While waiting for cardiac surgery in the intensive care unit, sudden clinical state worsening was observed with symptoms of cardiogenic shock such as tachycardia (HR 120-130 bpm), blood pressure decrease (BP 65/40 mm Hg) and logic contact impaired. The following echocardiographic imaging showed heart tamponade (Figure $1 \mathrm{E}$ ). The patient was prepared for emergency surgery. EuroSCORE II was calculated as $21.75 \%$. Surgery with arterial cannulation via femoral artery access to facilitate extracorporeal circulation was performed. The aneurysmal sac was cut to some degree over the right atrium to perform vein cannulation. In the next step tamponade was decompressed, showing ruptured pseudoaneurysmal sac size $51 \times 39 \times 31 \mathrm{~mm}$ (Figure $1 \mathrm{~F}$ ). Cooley's method was used to close the aneurysm. Clamp time was $39 \mathrm{~min}$ and whole extracorporeal circulation time was $79 \mathrm{~min}$. The patient was discharged from hospital on the $12^{\text {th }}$ day after surgery in a good clinical state.
Despite development of interventional cardiology and widespread use of endovascular procedures in treatment of acute coronary syndrome, there are still observed mechanical complications of myocardial infarction. Left ventricular pseudoaneurysm is a relatively rare complication that is reported in less than $0.1 \%$ of all $\mathrm{MI}$ patients and the main risk factors are older age, female sex, hypertension and inferior and lateral wall MI [1]. Pseudoaneurysm is formed when cardiac rupture is contained by adherent pericardium or scar tissue, in contrast to true aneurysm which comprises full thickness of the wall [1]. Around $40 \%$ of all left ventricular wall ruptures occurred during the first $24 \mathrm{~h}$ and $85 \%$ within the first week [2]. The most common localization of pseudoaneurysm is inferolateral (posterior) wall (43\%), lateral wall (28\%), then apex (24\%) followed by other segments at equal frequency [3]. Symptoms presented by patients with left ventricular pseudoaneurysm are varied and not specific, which makes the diagnosis challenging [2]. Both transthoracic and transesophageal echocardiography as well as CMR are used in the diagnostic process and in differentiating pseudoaneurysm from true aneurysm [2].

Most cases of this type of $\mathrm{MI}$ complications result in death due to cardiogenic shock because of heart tamponade. Knowing the natural course of acute heart pseudoaneurysm, medical therapy should be initiated urgently. The most effective method of treatment is surgical intervention, mostly open heart operation, although percutaneous coil embolization has been reported to be an alternative management in selected patients [4]. Nonetheless, the decision about the operation should be carefully analyzed because of the high surgical mortality rate [2].

\section{Corresponding author:}

Helena Krysztofiak, $1^{\text {st }}$ Department of Cardiology, Poznan University of Medical Sciences, Poznan, Poland,

e-mail: helenakrysztofiak@gmail.com

Received: 22.03.2020, accepted: 19.05.2020. 


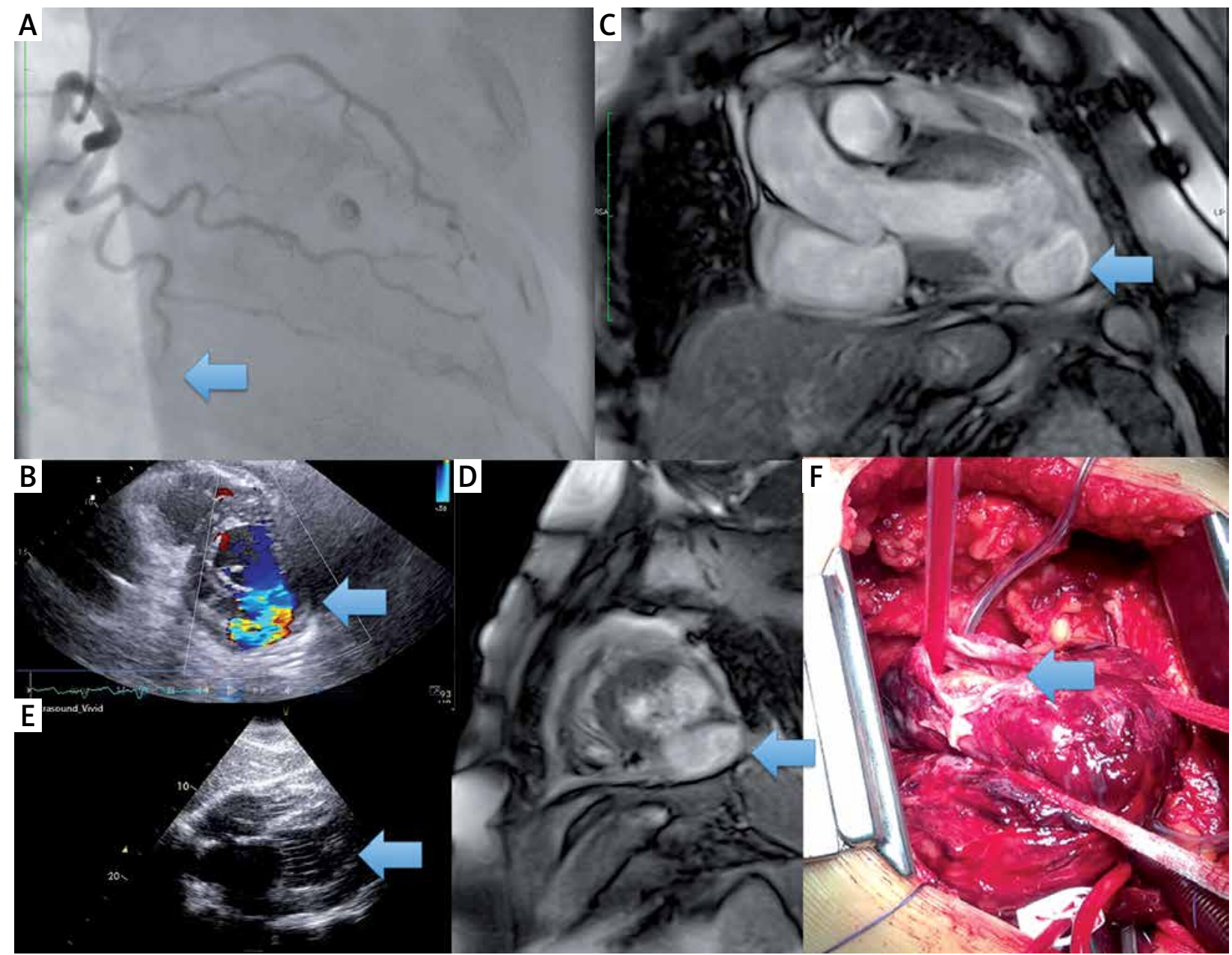

Figure 1. A - Coronary angiography showing occlusion of circumflex artery (LCX) in distal part. B - Echocardiography parasternal short axis-view, color Doppler imaging showing pseudoaneurysm of lateral wall of left ventricle and small pericardium effusion. Cardiac magnetic resonance (CMR) imaging confirmed pseudoaneurysm. CMR long axis-view (C) and short axis-view (D) showing pseudoaneurysm. E - Because of worsening of clinical status, while waiting for the urgent cardiac surgery, controlled echocardiography was performed, showing heart tamponade. F - Emergency surgery (EuroSCORE II - 21.75\%) in extracorporeal circulation with arterial cannulation via femoral artery access was performed. Cooley's method was used to close the aneurysm

\section{Conflict of interest}

The authors declare no conflict of interest.

\section{References}

1. Alapati L, Chitwood WR, Cahill J, et al. Left ventricular pseudoaneurysm: a case report and review of the literature. World I Clin Cases 2014; 2: 90-3.

2. Avegliano G, Conde D, Ruiz MI, et al. Lateral left ventricular wall rupture following acute myocardial infarction: pathophysiological interpretation by multimodality imaging approach. Echocardiography 2014; 31: E296-9.

3. Frances C, Romero A, Grady D. Left ventricular pseudoaneurysm. J Am Coll Cardiol 1998; 32: 557-61.

4. Li Y, Deng L, Song Y. Surgical treatment of huge left ventricular outflow tract pseudoaneurysm. Interact Cardiovasc Thorac Surg 2018; 27: 613-4. 